\title{
Face Detection using Fuzzy Skin Color Segmentation
}

\author{
Sabiha Sultana \\ Department of CSE \\ Jahangirnagar University \\ Savar, Dhaka-1342, \\ Bangladesh
}

\author{
Md. Saiful Islam \\ Department of CSE \\ Jahangirnagar University \\ Savar, Dhaka-1342, \\ Bangladesh
}

\author{
Md. Golam Moazzam \\ Department of CSE \\ Jahangirnagar University \\ Savar, Dhaka-1342, \\ Bangladesh
}

\begin{abstract}
This paper presents a reliable method for detecting human faces in color images. The system is based on the visual information of the face and is commenced with the detection of face regions by connected component analysis of the skin color segmentation of images in $\mathrm{HSV}, \mathrm{YC}_{\mathrm{B}} \mathrm{C}_{\mathrm{R}}$, YIQ color model and the Fuzzy membership function. The Fuzzy Membership function is applied to search for possible face regions in an image. The proposed method is robust in that it can deal with illumination changes and moderate rotations.
\end{abstract}

\section{Keywords}

Fuzzy Inference, Mamdani Style, Color Model, Membership Function, Skin Color.

\section{INTRODUCTION}

Automatic face detection in a cluttered image is an important step for face recognition system. Human face detection is difficult because there can be huge variations in the appearance of face patterns and many of these variations are difficult to parameterize. The starting problem in most of the cases of facial feature extraction is face detection, which deals with locating faces in images [1], [2].

In this paper, a reliable method for detecting human faces in color images is introduced. The location of the face regions is detected using the Fuzzy membership function. The Fuzzy Membership function is applied to search for possible face regions in an image. The proposed method is robust in that it can deal with illumination changes and moderate rotations.

\section{PROPOSED METHODOLOGY}

Face detection is one of the visual tasks which humans can do effortlessly. However, in computer vision terms, this task is not easy. The great challenge for the face detection problem is the large number of factors that govern the problem space. The long list of these factors include the pose, orientation, facial expressions, facial sizes found in the image, luminance conditions, occlusion, structural components, gender, human race, the scene and complexity of image's background [3]

Although a large number of face detection methods have been presented in the literature, few approaches address more than one or two variables. They assumed their own problem constraints to limit the problem space instead of challenging this wide range of factors.

The major difficulty in designing a robust face detection system is the limitation of vision and understanding of a computing machine. The ability to understand that the given image is not just a collection of pixels but is of human face (if any) is truly incredible [4], [5]. Face detection is concerned with determining which part of an image contains face. This is the first step of face recognition which requires both high- and low-level visual and geometric information processing. This work presents Fuzzy membership function for detecting human faces in a complex background. Face detection is achieved by employing template matching between a known face image and the input image. The main steps employed for the face detection process in a color image is shown in Fig. 1.

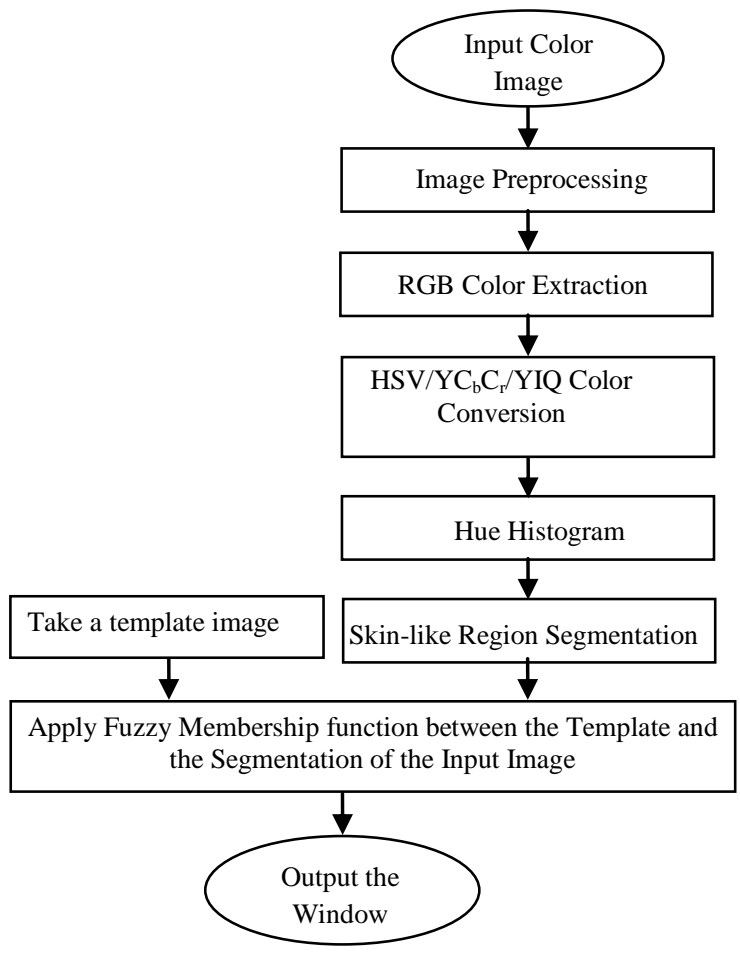

Fig. 1: Basic steps for face detection

The original image is obviously a color image. For detecting the face area, at first the image is processed using histogram equalization because, the face image may be of very poor contrast because of the limitation of the lighting conditions. Various sources of noise may also exist in the input image. So, low-pass filters are used to suppress the noise [6], [7]. The image content is then converted from $R G B$ to three different color models such as $H S V, Y C_{b} C_{r}$, and $Y I Q$ color space for face detection purpose.

To apply the Fuzzy membership function for face detection, a template of the face image obtained from averaging the hue value of pixels of a number of similar looking face images of several persons is constructed. The template face image is then moved through the whole image to find the location where the most suitable match exists. In order to determine whether the segmented region of the input image is a face or not, the membership value of the segmented region is computed by means of hue similarities for $H S V$ space.

Face detection is performed by using color images. The existence of facial features like eyes, nose, mouth, and so on are the evidences that the candidate region is indeed a face. First different color models are used to detect a candidate 
cluster as a face in an image using fuzzy membership function and then a face is detected.

For face detection the proposed method follows the following steps:

1. Input: $R G B$ image data

2. Convert $R G B$ image into $H S V, Y I Q, Y C_{b} C_{r}$ space model.

3. Select an upper and lower bounds for $C_{b}, C_{r}$ and $Y, I$ values. Pixels that lie between the upper and lower bounds are assigned to their membership values in the interval $[0,1]$, and pixels that are outside the bounds are set to the membership value 0 .

4. Detect possible face-like clusters using the method described in Step 3.

5. Select one cluster and apply fuzzy logic modeling approach to decide if the cluster contains a face or not (depending on whether facial features, such as, eyes, nose, mouth, eye-brow, etc. contains or not).

6. If all clusters are processed, then go to step 4, otherwise go to step 5 .

A number of experiments were carried out for various input color images with complex backgrounds. In every case, the experiments have shown very promising results. The highest detection rate of the system is approximately $96 \%$.of image processing and analysis methods that are involved in this thesis.

\section{FUZZY INFERENCE FOR FACE DETECTION}

The basic idea applied to problem solving for face detection is illustrated in this section.

\section{Fuzzy Logic}

A logic based on the two truth values True and False is sometimes inadequate when describing human reasoning. Fuzzy logic uses the whole interval between 0 (False) and 1 (True) to describe human reasoning. In this context, Fuzzy logic (FL) is a problem-solving control system methodology that lends itself to implementation in systems ranging from simple, small, embedded micro-controllers to large, networked, multi-channel PC or workstation-based data acquisition and control systems. It can be implemented in hardware, software, or a combination of both. FL provides a simple way to arrive at a definite conclusion based on vague, ambiguous, imprecise, noisy, or missing input information.

\section{Fuzzy Membership Functions}

The membership function is a graphical representation of the magnitude of participation of each input. So the function that ties a number to each element $\mathrm{x}$ of the universe is called the membership function $\mu(x)$. A membership function is bellshaped (also called a $\pi$-curve), s-shaped (called an s-curve), a reverse s-curve (called z-curve), triangular, or trapezoidal [8].

A cosine function can be used to generate a variety of membership functions. The $s$-curve can be implemented as

$$
s\left(x_{l}, x_{r}, x\right)=\left\{\begin{array}{lc}
0, & x<x_{l} \\
\frac{1}{2}+\frac{1}{2} \cos \left(\frac{x-x_{r}}{x_{r}-x_{l}} \pi\right), & x_{l} \leq x \leq x_{r} \\
1, & x>x_{r}
\end{array}\right\}
$$

Where $x_{l}$ is the left breakpoint, and $x_{r}$ is the right breakpoint. The $z$-curve is just a reflection,

$$
z\left(x_{l}, x_{r}, x\right)=\left\{\begin{array}{ll}
1, & x<x_{l} \\
\frac{1}{2}+\frac{1}{2} \cos \left(\frac{x-x_{l}}{x_{r}-x_{l}} \pi\right), & x_{l} \leq x \leq x_{r} \\
0, & x>x_{r}
\end{array}\right\}
$$

The membership function employed for the fuzzy logic, is a $\pi$ - shaped curve, implemented as a combination of s- curve and z-curve, respectively. Standard Membership functions are shown in Fig. 2.

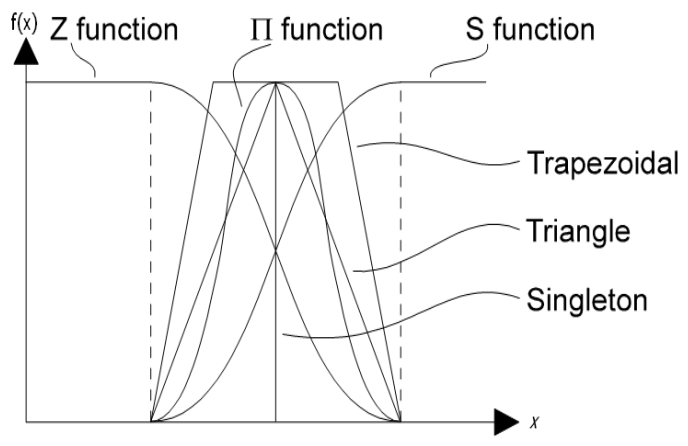

Fig. 2: Standard Membership Functions.

\section{Fuzzy Inference}

Inference is a technique by which, a given set of facts or postulates or axioms or premises $F_{1}, F_{2}, \ldots, F_{n}$, a goal $G$ is to be derived. The process of reasoning based on Fuzzy logic is called fuzzy inference. For example,

$$
\begin{aligned}
& \text { If the color is more_or_less_skin_like, } \\
& \text { Then image_contains face. }
\end{aligned}
$$

\section{Fuzzy Rule}

A conditional statement in the form: IF $x$ is $A$ THEN $y$ is $B$, where $x$ and $y$ are linguistic variables, and $A$ and $B$ are linguistic values determined by fuzzy sets.

- Rule 1: IF ( $C_{b}$ is extremely skin like) and $(\mathrm{Cr}$ is more_or_less skin like) then (Image is Face_like)

- Rule 2: IF ( $C_{b}$ is not_so skin like) and $(\mathrm{Cr}$ is more_or_less skin like) or ( $Y$ is extremely low)then (Image is Not_Face_Like)

Inputs to a fuzzy system can be

- fuzzy, e.g. (Color_Value $=$ Moderate), defined by membership functions

- exact, e.g.: $\left.\left(C_{b}=100\right) ; C_{r}=80\right)$, defined by crisp values.

Outputs from a fuzzy system can be

- fuzzy, i.e. a whole membership function, or

- exact, i.e. a single value is produced on the output.

\section{Mamdani-Style Inference}

The Mamdani-Style inference rule is as follows:

Rule 1:

IF $x$ is $\mathrm{A} 3$

OR $y$ is $\mathrm{B} 1$

THEN $z$ is $\mathrm{C} 1$
Rule 1:

IF hue is extremely skin_like

OR saturation is good

THEN image is face_like 
Rule 2:

IF $x$ is $\mathrm{A} 2$

AND $y$ is B2

THEN $z$ is $\mathrm{C} 2$

Rule 3:

IF $x$ is A1

THEN $z$ is $\mathrm{C} 3$

Rule 2:

IF hue is marginally skin_like

AND saturation is very poor

THEN image is a_little_bit face_like

Rule 3:

IF hue is inadequate

THEN image is not_face_like

\section{A: Fuzzy Rules for Skin Color Detection in $\mathrm{YC}_{b} C_{r}$ Color Model (Mamdani-Style Inference)}

The fuzzy logic rules applied for skin detection are as follows [9], [10]:

1. IF $\mathrm{Cb}$ is Extremely skin like And $\mathrm{Cr}$ is Extremely skin like THEN the pixel is white.

2. IF $\mathrm{Cb}$ is Extremely skin like And $\mathrm{Cr}$ is Skin like THEN the pixel is white

3. IF $\mathrm{Cb}$ is Extremely skin like And $\mathrm{Cr}$ is Morl skin like THEN the pixel is white

4. IF $C b$ is Skin like And $C r$ is Extremely Skin like THEN the pixel is white

5. IF $\mathrm{Cb}$ is Skin like And $\mathrm{Cr}$ is Skin like THEN the pixel is black

6. IF $\mathrm{Cb}$ is Skin like And $\mathrm{Cr}$ is Morl skin like THEN the pixel is black

7. IF $\mathrm{Cb}$ is Morl skin like And $\mathrm{Cr}$ is Extremely skin like THEN the pixel is white

8. IF $C b$ is Morl skin like k And $C r$ is Skin like THEN the pixel is black

9. IF $\mathrm{Cb}$ is Morl skin like And $\mathrm{Cr}$ is Morl skin like THEN the pixel is white

\section{B: Fuzzy Rules for Skin Color Detection in YIQ Color Model (Mamdani-Style Inference)}

The fuzzy logic rules applied for skin detection are as follows [9], [10]:

1. IF $Y$ is Extremely skin like And $I$ is Extremely skin like THEN the pixel is white.

2. IF $Y$ is Extremely skin like And $I$ is Skin like THEN the pixel is white

3. IF $Y$ is Extremely skin like And $I$ is morl skin like THEN the pixel is white

\section{Membership Function by Fuzzy Rules}

The membership function employed for different $C_{b}$ and $C_{r}$ values in order to detect face color segmentation on the basis of the fuzzy rules are, as shown in Fig. 3.

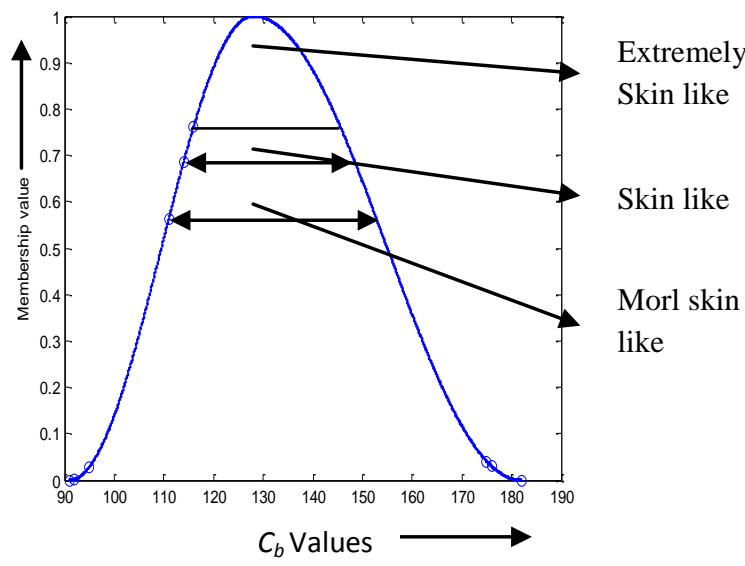

Fig. 3: Membership function for skin color segmentation
4. IF $Y$ is Skin like And $I$ is Extremely skin like THEN the pixel is white

5. IF $Y$ is Skin like And $I$ is Skin like THEN the pixel is black

6. IF $Y$ is Skin like And $I$ is Morl skin like THEN the pixel is black

7. IF $Y$ is Morl skin like And $I$ is Extremely Skin like THEN the pixel is white

8. IF $Y$ is Morl skin like $\mathrm{k}$ And $I$ is Skin like THEN the pixel is black

9. IF $Y$ is Morl skin like And $I$ is Morl skin like THEN the pixel is white

\section{C: Fuzzy Rules for Skin Color Detection in HSV Color Model (Mamdani-Style Inference)}

The fuzzy logic rules applied for skin detection are as follows [9], [10]:

1. IF $H$ is Extremely skin like And $S$ is Extremely skin like THEN the pixel is white.

2. IF $H$ is Extremely skin like And $S$ is Skin like THEN the pixel is white

3. IF $H$ is Extremely skin like And $S$ is morl skin like THEN the pixel is white

4. IF $H$ is Skin like And $S$ is Extremely skin like THEN the pixel is white

5. IF $H$ is Skin like And $S$ is Skin like THEN the pixel is black

6. IF $H$ is Skin like And $S$ is Morl skin like THEN the pixel is black

7. IF $H$ is Morl skin like And $S$ is Extremely Skin like THEN the pixel is white

8. IF $H$ is Morl skin like $\mathrm{k}$ And $S$ is Skin like THEN the pixel is black

9. IF $H$ is Morl skin like And $S$ is Morl skin like THEN the pixel is white

In the same way like as the above figure, membership can be calculated for different $\mathrm{C}_{\mathrm{r}}, \mathrm{Y}, \mathrm{I}, \mathrm{H}$ and $\mathrm{S}$ values.

\section{EXPERIMENTAL RESULTS}

In experiments face images are analyzed to demonstrate the feasibility of the proposed method. The effectiveness and robustness of this approach are justified using different images with various kinds of expressions. Experiments are carried out on Intel(R) Core(TM) i5-3470 CPU @ 3.20GHz PC with 4.00 GB RAM. The algorithm has been implemented using Visual $\mathrm{C}++$. When a complex image is subjected to the input, the face detection result highlights the facial part of the image. Images of different persons are taken at their own work places and at different environments both in shiny and gloomy weather. Most of the images are taken using a digital camera, but some are from scanner. The algorithm is capable of detecting single face in an image. For multiple faces, the system finds the dominant face only, i.e. the face containing more number of skin colored pixels. A total of 273 images, including more than 76 different persons, are used to investigate the capacity of the proposed algorithm for different color model. Experimental results demonstrate that 
the success rate of above $96 \%\left(\frac{265}{273} \times 100 \%=97.06 \%\right)$ is

achieved for $Y C_{b} C_{r}$ color model. The main reason behind the failure of those images in finding face regions are occlusion and side view of faces, etc.

The experimental results are analyzed below for different colored input images (single face image and multiple face images) under different environment and lighting conditions.

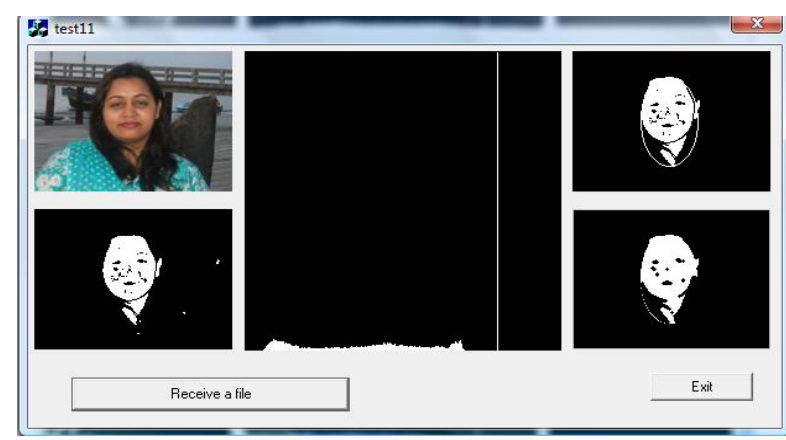

a) Single Face Images using HSV Color Model

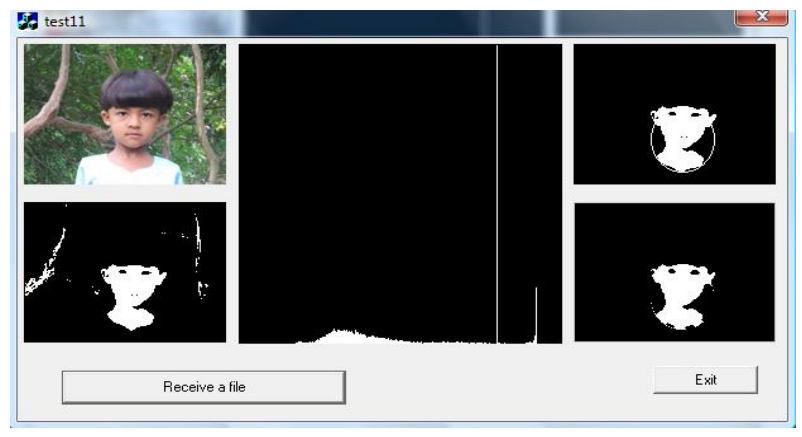

b) Single Face Images using YCbCr Color Model

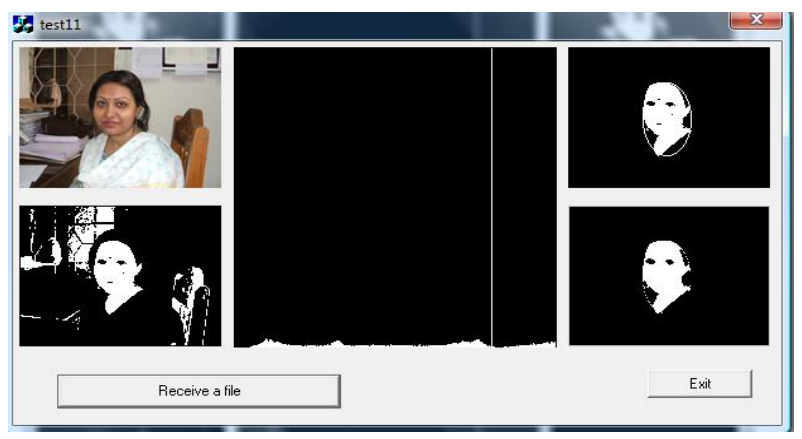

c) Single Face Images using YIQ Color Model

Fig. 4: Single Face Images with Pose Variations

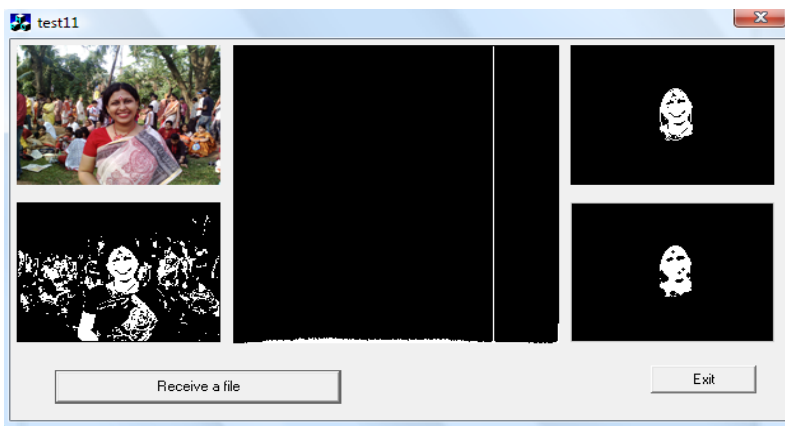

a) Multiple Face Images using HSV Color Model

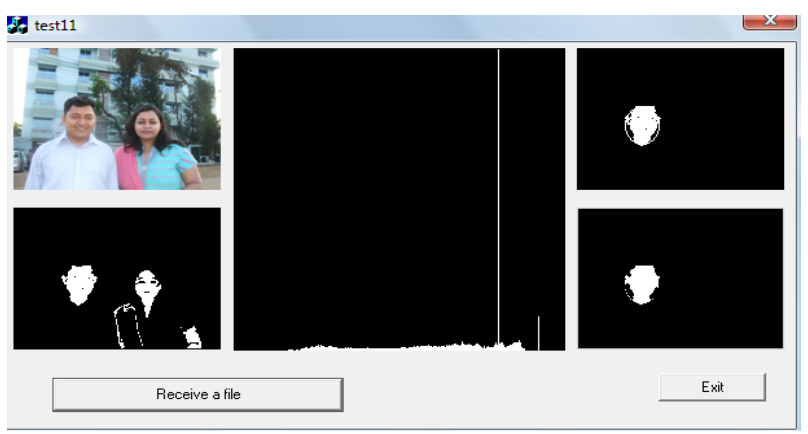

b) Multiple Face Images using YCbCr Color Model

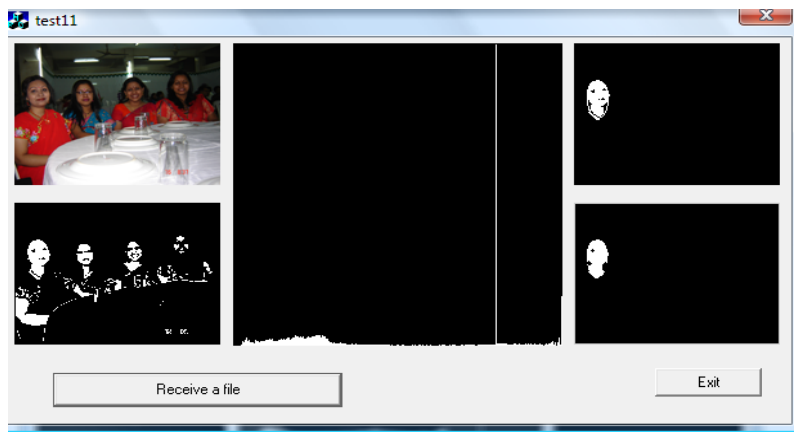

c) Dark face images using YIQ Color Model

Fig. 5: Multiple Face Images with Pose Variations

\section{PERFORMANCE ANALYSIS}

In this paper, face detection has been implemented using Fuzzy membership function to search for the face of a particular individual in an image. The effectiveness of the face detection algorithm has been tested both in simple and complex backgrounds for different types of face and non-face images of $160 \times 120$ resolution. These images are taken under different environment: some are under sunny environment; some are under cloudy environment; some are dark images; some are bright images, and so on. In all cases, experiments have shown promising results. Table I summarizes the performance analysis for Fuzzy membership based face detection under all possible environments for $Y C_{b} C_{r}$ color model. 
Table I: Performance analysis for Fuzzy membership based face detection for $\mathrm{YC}_{\mathrm{b}} \mathrm{C}_{\mathrm{r}}$ color model

\begin{tabular}{|c|c|c|c|c|}
\hline \multicolumn{2}{|c|}{$\begin{array}{c}\text { Image } \\
\text { Condition }\end{array}$} & $\begin{array}{c}\text { No. of } \\
\text { Images }\end{array}$ & $\begin{array}{c}\text { Correctly } \\
\text { Detected }\end{array}$ & $\begin{array}{c}\text { Success } \\
\text { Rate }\end{array}$ \\
\hline \multirow{2}{*}{$\begin{array}{c}\text { Outdoor } \\
\text { Images }\end{array}$} & Sunny & 55 & 54 & $98 \%$ \\
\cline { 2 - 5 } & Cloudy & 50 & 48 & $96 \%$ \\
\cline { 2 - 5 } & Dark & 40 & 38 & $95 \%$ \\
\hline \multirow{2}{*}{$\begin{array}{c}\text { Indoor } \\
\text { Images }\end{array}$} & Bright & 50 & 49 & $98 \%$ \\
\cline { 2 - 5 } & Dark & 41 & 39 & $95 \%$ \\
\hline \multicolumn{2}{|r|}{$\begin{array}{c}\text { Images with } \\
\text { Occlusion }\end{array}$} & 37 & 34 & $92 \%$ \\
\hline \multicolumn{2}{|r|}{}
\end{tabular}

It is observed that among 273 face images only 8 faces are found false and the maximum success rate of the implemented system is $98 \%$ under sunny (Outdoor) and bright (Indoor) environments. Thus, the overall success rate of above $96 \%$ is achieved in this method.

Experiments are performed using different color model and Fuzzy membership function. Table II summarizes the performance analysis for Fuzzy membership based face detection under all color models.

Table II: Performance analysis for Fuzzy membership based face detection using three color models

\begin{tabular}{|c|c|c|c|}
\hline $\begin{array}{c}\text { Color } \\
\text { Model }\end{array}$ & $\begin{array}{c}\text { No. of } \\
\text { Images }\end{array}$ & $\begin{array}{c}\text { Correctly } \\
\text { Detected }\end{array}$ & $\begin{array}{c}\text { Average Success } \\
\text { Rate }\end{array}$ \\
\hline$H S V$ & 260 & 245 & $94 \%$ \\
\hline$Y I Q$ & 260 & 248 & $95 \%$ \\
\hline$Y C_{b} C_{r}$ & 273 & 265 & $96 \%$ \\
\hline
\end{tabular}

Experimental results show that the algorithm is capable of detecting the faces in color images with different backgrounds. It can detect a rotated human face, even if the face is under shadow, wearing glasses, or under bad lighting conditions.

\section{CONCLUSIONS}

The area of face detection has attracted considerable attention in the advancement of human-machine interaction as it provides a natural and efficient way to communicate between humans and machines. Although human beings accomplish these tasks countless times a day, they are still very challenging for machine vision. Most of the researchers attack this kind of problem with face localization and feature selection with frontal view faces and without facial expression and normal lighting conditions although the variation between the images of the same face is too large due to facial expression, hair style, pose variation, lighting conditions, make-up, etc.

This paper proposes a face detection method for color images in the presence of varying lighting conditions as well as complex backgrounds. The location of the face region is detected using the Fuzzy Membership function. The Fuzzy Membership function is applied to search for possible face regions in an image. Experimental results demonstrate that this face detection process works predictably reliable, and regardless of the perspective.

Some achievements of this work are described below.

- The proposed method has been successfully examined with color images.
- With more than 273 images, our method is successfully tested with various types of faces affected by intensity, occlusion, structural components, facial expression, illumination, noise, and orientation. It can detect above $96 \%$ of the faces with an acceptable number of false detections.

- For multiple face images the system finds the dominant face, i.e. the face containing more number of skin colored pixels.

- This method can reliably detect faces that vary in viewpoint from frontal to side view. Previously only frontal face detection had been demonstrated reliably.

\section{REFERENCES}

[1] M. H. Yang, D. Kriegman, and N. Ahuja, "Detecting Faces in Images: A Survey", IEEE Transaction on Pattern Analysis and Machine Intelligence, Vol. 24, No. 1, January 2002.

[2] M. A. Bhuiyan, "Face Detection and Facial Feature Extraction", 4th International Conference on Computer and Information Technology, University of Dhaka, Bangladesh, Vol. 1, No. 11, pp. 110-115, 2001.

[3] Hielmas, Erik; Low, Boon Kee; "Face detection: A survey", Computer Vision and Image Understanding, Vol. 83, No. 3, p. 236-274, 2001.

[4] Rafael C. Gonzalez and R. E. Woods, "Digital Image Processing", Pearson Education Asia, Second Edition, 2002.

[5] A. Rosenfeld and A. C. Kak, "Digital Image Processing", Vols. I and II, New York: Academic Press, 1982.

[6] Yang, G.; Huang, Thomas; "Human face detection in a complex background, Pattern Recogniton, Vol. 27, No. 1, p. 53-63, 1994.

[7] Freeman, William; Pasztor, Egon; Carmichael, Owen; "Learning low level vision", Int. Journal of Computer Vision, Vol. 40, No. 1, p. 25-47, 2000.

[8] Rowley, Henry; Beluga, Shumeet; Kanade, Takeo; "Neural network-based face detection", IEEE Transactions on Pattern Analysis and Machine Intelligence, Vol. 20, No. 1, pp. 23-37, 1998.

[9] S.Y. Lee, Y.K. Ham, R.H. Park, "Recognition of human front faces using knowledge-based feature extraction and neuro-fuzzy algorithm, Pattern Recognition, Vol. 29, No. 11, p. 1863-1876, 1996.

[10] T. S. Huang (ed.), "Picture Processing and Digital Filtering", In topics in Applied Physics, Vol. 6, Berlin: Springer-Verlag, 1975.

\section{AUTHOR'S PROFILE}

Sabiha Sultana completed her B.Sc (Hons) in Electronics and Computer Science from Jahangirnagar University in 1998 and M.S. in Computer Science and Engineering from the same University in 2001, respectively. She is now an Assistant Professor in the Dept. of Computer Science and Engineering, Dhaka City College, Dhaka, Bangladesh. Her research interests include Digital Image Processing, Artificial Intelligence, Computer Graphics, Algorithm Design, Neural Networks, Computer Vision Object Oriented Programming and so on. 
Md. Saiful Islam completed his B.Sc (Hons) in Electronics and Computer Science from Jahangirnagar University in 1998. He is now a programmer in Bangladesh Public Administration Training Center, Savar, Dhaka, Bangladesh. His research interests include Web Programming, Digital Image Processing, Artificial Intelligence, Computer Graphics, Neural Networks, Computer Vision and so on.

Md. Golam Moazzam completed his B.Sc (Hons) in Electronics and Computer Science from Jahangirnagar
University in 1997 and M.S. in Computer Science and Engineering from the same University in 2001, respectively. $\mathrm{He}$ is now an Associate Professor in the Dept. of Computer Science and Engineering, Jahangirnagar University, Dhaka, Bangladesh. His research interests include Digital Image Processing, Artificial Intelligence, Computer Graphics, Neural Networks, Computer Vision and so on. 